\title{
Proporciones de debris en réplicas de talla experimental
}

\author{
José Manuel Maillo Fernánoez ${ }^{1}$
}

\begin{abstract}
RESUMEN
En este trabajo se presentan los resultados de una serie de réplicas de

talla para conocer la dinámica de la fracción debris. El resultado obtenido es una curva de distribución característica en la que a menor longitud se obtiene mayor número de restos. Las conclusiones pueden ser utilizadas en

los análisis post-deposicionales de yacimientos arqueológicos.
\end{abstract}

\section{PALABRAS CLAVE}

Debris, tipometria, réplicas de talla, análisis post-deposicionales.

\section{ABSTRACT}

In this paper we present a series of experiments to investigate the smallsize debitage dynamic. The results of the experiments suggest an ideal layout. The results should be used in formation processes of the archaeological record.

\section{KEY WORDS}

Debris, tipometry, lithic replic, post-deposicional analisys.

\section{INTRODUCCIÓN}

La recuperación sistemática y exhaustiva del registro arqueológico es cada vez más habitual en las excavaciones de nuestro país. Sin embargo, los restos recuperados no son tratados de igual forma, siendo algunos de ellos recogidos y estudiados de forma testimonial. Es este el caso del debris

1 Becario F.P.U. Dpto. Prehistoria e Historia Antigua. UNED. 
al que hay que unir generalmente su pequeño tamaño y su clasificación en una segunda categoría dentro de los estudios tecnológicos, lo que hace a este tipo de objeto arqueológico poco atractivo para los tecnólogos, aunque son, sin duda, los restos líticos más numerosos en los conjuntos paleolíticos.

En este trabajo presentamos los resultados de un experimento realizado con debris con la finalidad de hallar las proporciones en las que sus diferentes tipos aparecen a partir de réplicas de retoque y, por otro lado, se muestra la distribución métrica de los mismos. Los resultados de este estudio se pueden aplicar a yacimientos arqueológicos para poder llegar a discernir posibles procesos postdeposicionales.

Consideramos como debris a los fragmentos de pequeño tamaño que componen el sustrato de muchas industrias (Bernaldo de Quirós et al.1980). Dividiendo éstos en tres grupos:

a) Debris de lascado: lascas o fragmentos de pequeño tamaño producto de la acción de tallar en los que se pueden ver algunas de las características producidas por la talla (cara bulbar, extracciones en el reverso, talón...).

b) Debris de astillado: son fragmentos irreconocibles de materia prima producidos en la actividad de talla pero que no son producidos por acción directa del percutor, sino por las tensiones del golpe de talla sobre la materia prima. Su forma puede ser variada de formas piramidales a cúbicas.

c) Debris térmico: son los fragmentos producidos por la alteración térmica de la materia prima (sólo en el sílex). Son de dos tipos, los primeros con forma abombada en su cara bulbar y con planta circular y los segundos con planos rugosos y morfología irregular.

\section{ANTECEDENTES}

La fracción debris es, sin duda, el tipo de resto lítico más numeroso de los que se recuperan en los yacimientos paleolíticos (Freeman et alli, 1998, Roebroeks et al., 1997). Además, es el único que no sufre modificaciones por cuestiones antrópicas, lo que le confiere una gran importancia tafonómica. Los estudios sobre debris no son demasiado numerosos, pero sí significativos. Los investigadores han repartido sus esfuerzos en dos campos diferentes:

1. Análisis tecnológico.

2. Análisis espacial. 
Análisis tecnológico

En este apartado las escasa investigaciones se han centrado en vislumbrar aspectos cualitativos y cuantitativos mediante réplicas de talla (Henry et alli, 1979; Patterson y Sollberger, 1978; Baumler y Downum, 1989, Shott, 1994) y análisis de los debris producidos. En estos trabajos se tienen en cuenta variables como el tamaño, grosor, peso, etc. Sin embargo, existen graves contradicciones en los estudios de este tipo. En unos casos la materia prima no está controlada, en otros los experimentos de talla son bien resueltos en laboratorio, pero en el registro arqueológico se produce la mezcla de todos ellos en mayor o menor proporción por lo que las conclusiones resultan demasiado banales al no poder ser sometidas a la evidencia del registro fósil.

Dentro de este apartado destaca otro tipo de estudios común en lascas y hojas como es el de los remontajes (Allard, 1993). En este caso se llega a crear el molde de una punta solutrense gracias al remontaje de sus debris y además ver los modos de utilización del espacio dentro del yacimiento.

\section{Análisis espacial}

Estos estudios resultan también escasos en la bibliografía y todos ellos siguen una línea metodológica similar. El objetivo principal en este tipo de trabajos es estudiar posibles patrones que permitan observar diferencias entre depósitos primarios y secundarios (Behm, 1983; Baumler, 1985). Ambos realizan experimentos sobre la incidencia de agentes naturales sobre los debris que se encuentran en los yacimientos. En todos los experimentos realizados la fuerza natural que actúa sobre el yacimiento (en este caso la lluvia) no está controlada.

Un método novedoso es el que propone el estudio de la industria lítica dentro de parámetros sedimentológicos (Fladmark, 1982). En este tipo de estudios se trabaja con la fracción menor a $1 \mathrm{~mm}$ y que se autor denomina microdebitage. Sus resultados son muy interesantes ya que entre un $80-90 \%$ de los debris que se producen en la talla están dentro de estas dimensiones. Trabajos posteriores realizados con esta metodologia (Hull, 1987), proporcionan datos reveladores sobre la formación de yacimientos. El problema esencial de este tipo de estudios es que los procesos postdeposicionales afectan en mayor intensidad a esta fracción lítica, aunque sean de muy baja intensidad. Por otro lado resulta sospechoso como son manipulados los datos en algún trabajo (Hull, 1987) para conseguir los resultados que ya aportan otros tipos de estudios. 
Siguiendo la misma metodología pero con miras más amplias encontramos otro trabajo (Stein, 1987; Stein y Telster, 1989). Aquí se estudian todos los restos arqueológicos posibles (piedra, metal, cerámica, etc.) llegando a conclusiones tafonómicas muy interesantes.

\section{EXPERIMENTACIÓN}

Este experimento es un estudio piloto para conocer las proporciones métricas de la fracción debris en réplicas de talla. Esto permitirá poder discernir posibles alteraciones post-deposicionales de baja-media intensidad en yacimientos arqueológicos. Para ello se han realizado una serie de 15 réplicas de retoque. Los resultados serán utilizados en un estudio sobre la industria lítica del yacimiento de La Carihuela (Piñar, Granada) en proceso de estudio.

\section{a) Metodología}

Para el experimento se ha utilizado sílex de dos procedencias diferentes. Por un lado tres tipos de sílex de los más comunes en el yacimiento de La Carihuela (Vega, 1988):

1. Sílex tabular denso (S2). Se presenta en tabletas prismáticas y de interior bandeado, se puede encontrar enfrente de la cueva, en los alrededores del pueblo de Pínar.

2. Calcedonia negra o gris oscuro (S3): Se encuentra en nódulos esféricos de pequeño y mediano tamaño. Es frecuente en los glacis de la zona (Iznalloz y Sierra Harana), pero también se encuentra a 100 metros de la cueva.

3. Calcedonias anaranjadas y sílex jaspeados (S5): Se encuentran en nódulos grandes y de gran calidad al sur de la cueva.

Por otro lado sílex de la zona de Vallecas (Madrid) para controlar la variable de la materia prima. Ésta es un problema importante en otros trabajos de esta índole, como ya se ha visto.

El retoque de las piezas se ha realizado mediante percusión directa con percutor duro (cuarcita). De las 15 réplicas, las cinco primeras pertenecen al sílex de Vallecas ( $\left.n .{ }^{2} 1-5\right)$, mientras que el resto (n. $\left.{ }^{\circ} 6-15\right)$ corresponden a los diferentes sílex de la cueva de La Carihuela. El proceso de obtención de la muestra a estudiar es el siguiente: talla de la pieza y recogida minuciosa de todos los restos generados, toma de medidas (largo $x$ ancho) y clasificación de los mismos en debris de astillado o lascado. 


\section{b) Resultados}

Observando los datos de la tabla 1 se puede apreciar que en los intervalos de menor tamaño los debris se encuentran bien representados, mientras según se avanza en tamaño esta disminuye hasta desaparecer en muchos de los intervalos. Esta distribución genera una curva bien conocida en la bibliografía (Schick, 1986), con un marcado sentido descendente hasta longitudes de 6-9 $\mathrm{mm}$ donde desciende y se presenta constante y poco representada (gráfico 1). Este tipo de curva es la que se observa en todos los experimentos, sin tener en cuenta los porcentajes, sino la distribución entre ellos, excepto en el experimento n. ${ }^{1} 1$ en los que los dos primeros intervalos presentan porcentajes similares. En el resto esta diferencia entre el primer intervalo y el segundo queda muy marcada.

Si estudiamos los experimentos discriminando la materia prima se observa que en todos los casos ésta no es condicionante de los resultados. Los datos presentan una distribución que no es característica en

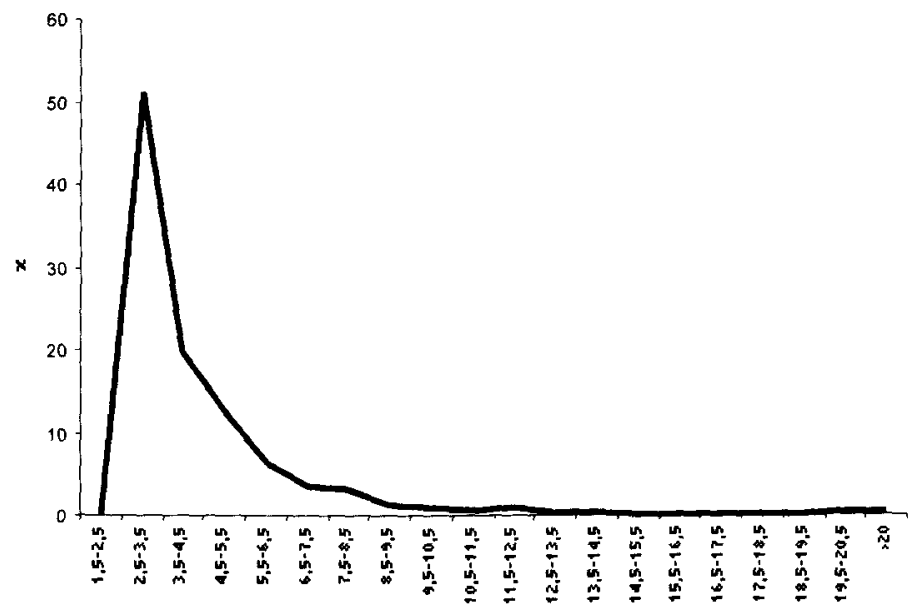

Gráfico 1. Curva modelo resultante de la experimentación.

sus porcentajes de un tipo u otro de sílex empleado. Por otro lado, el tipo de retoque tampoco representa un condicionante para que la curva presentada se produzca; al menos en el caso del sílex del tipo $S 2$ y el de Vallecas el único en el que hay variedad de retoque en el experimento. 


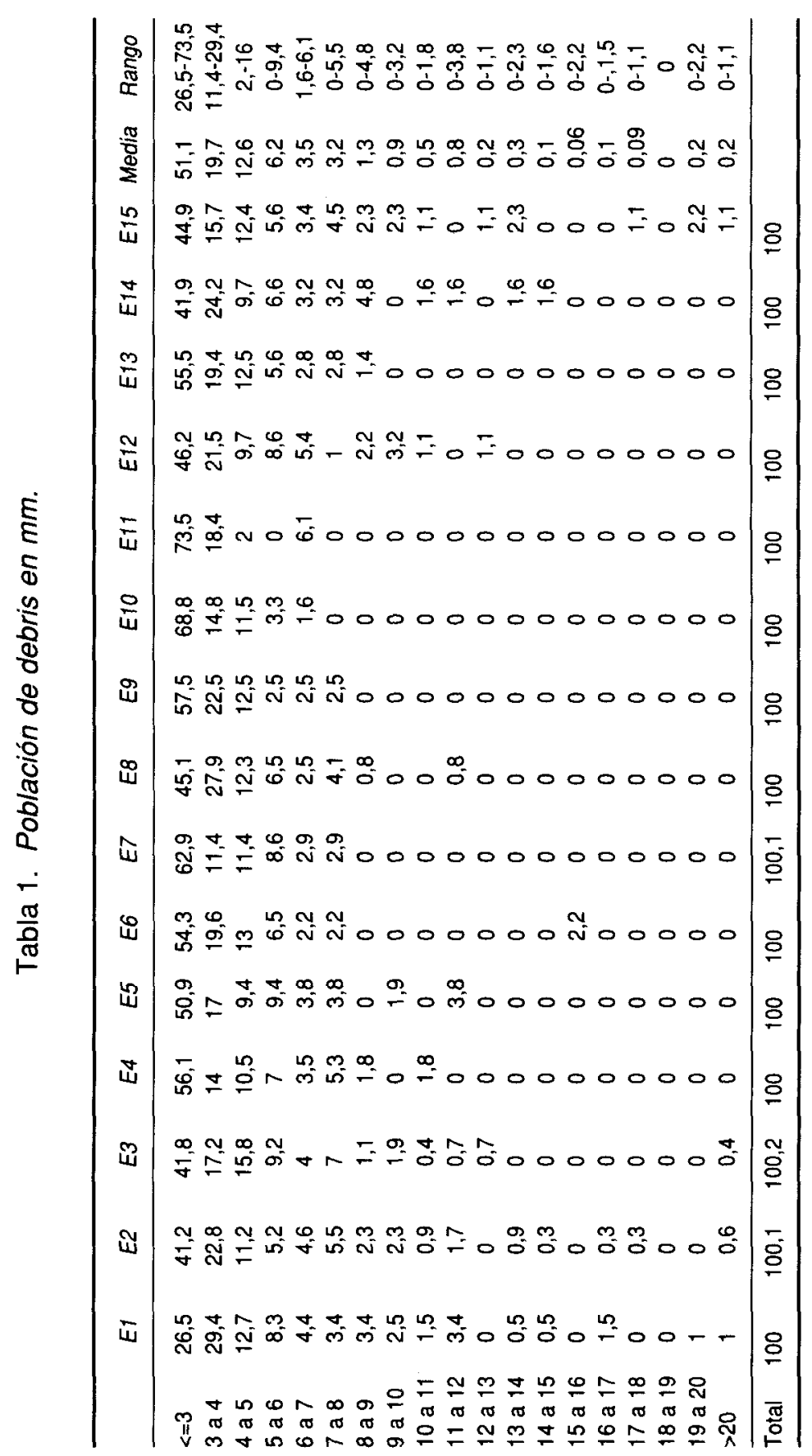


Otro aspecto a tener en cuenta en este estudio es el tipo de debris generado. El motivo de esto es que los debris de astillado que aparecen en grandes porcentajes, pueden estar producidos por alteraciones post-deposicionales. Para tener controlada esta posible variable se han clasificado los debris en dos tipos (lascado y astillado) y se han obtenido los porcentajes de ambos. Los resultados se pueden consultar en las tablas 3 y 4 , por lo que estudiando dichas tablas podemos dar un porcentaje de un $15 \%$ a la relación debris de astillado/debris de lascado (DA/DL) como el normal en actividades de talla, por lo que un porcentaje mayor de esta relación puede estar relacionado con una alteración postsedimentaria.

Tabla 2. Datos adicionales de los experimentos.

\begin{tabular}{rccccr}
\hline N. ${ }^{-}$ & Tipo de silex & $\begin{array}{c}\text { Dimensiones } \\
(\mathrm{mm})\end{array}$ & $\begin{array}{c}\text { Filo retocado } \\
(\mathrm{mm})\end{array}$ & Tipo de retoque & $\begin{array}{c}\text { Peso } \\
(\mathrm{gr})\end{array}$ \\
\hline 1 & $60-62-23$ & 9 & Simple-Profundo & 75 \\
2 & $73-45-19$ & 64 & Simple-Profundo & \\
3 & & $52-33-13$ & 45 & Simple-Profundo & 15 \\
4 & & $57-30-17$ & 43 & Quina-Profundo & 15 \\
5 & & $54-32-11$ & 43 & Simple-Profundo & 10 \\
6 & $\mathrm{~S} 3$ & $43-38-11$ & 45 & Simple-Profundo & 13 \\
7 & $\mathrm{~S} 3$ & $53-41-22$ & 40 & Simple-Profundo & 30 \\
8 & $\mathrm{~S} 3$ & $55-25-12$ & 50 & Simple-Marginal & 10 \\
9 & $\mathrm{~S} 2$ & $43-28-8$ & 43 & Simple (A)-Marginal & 5 \\
10 & $\mathrm{~S} 2$ & $40-43-12$ & 40 & Simple-Marginal & 15 \\
11 & $\mathrm{~S} 5$ & $58-46-24$ & 55 & Simple-Profundo & 25 \\
12 & $\mathrm{~S} 5$ & $38-40-9$ & 70 & Simple(A)-Profundo & 5 \\
13 & S2 & $47-38-17$ & 45 & Quina-Profundo & 20 \\
14 & S2 & $66-33-22$ & 39 & Quina-Profundo & 25 \\
15 & S2 & & & & \\
\hline
\end{tabular}

Tanto la materia prima como el tipo de retoque han podido influir en estos porcentajes. Observando las tablas 2,3 y 4, vemos como ninguna de las dos interfiere a la hora de crear un tipo u otro de debris y que la producción de éstos es aleatoria.

Tabla 3. Relación $D A / D L$ en silex procedente de Vallecas.

\begin{tabular}{ccccc}
\hline$N^{o}$ Experimento & $N^{o} D L$ & $N^{o} D A$ & Total & $\% D A$ \\
\hline 1 & 176 & 28 & 204 & 13.7 \\
2 & 312 & 35 & 347 & 10 \\
3 & 230 & 43 & 273 & 15.7 \\
4 & 54 & 3 & 57 & 5.26 \\
5 & 47 & 6 & 53 & 11.3 \\
\hline
\end{tabular}


Tabla 4. Relación $D A / D L$ en silex de los alrededores de La Carihuela.

\begin{tabular}{ccccc}
\hline$N^{\circ}$ Experimento & $N^{o} D L$ & $N^{\circ} D A$ & Total & $\% D A$ \\
\hline 6 & 40 & 6 & 46 & 13 \\
7 & 32 & 3 & 35 & 8.5 \\
8 & 110 & 12 & 122 & 9.8 \\
9 & 36 & 4 & 40 & 10 \\
10 & 57 & 4 & 61 & 6.5 \\
11 & 44 & 5 & 49 & 10 \\
12 & 87 & 6 & 93 & 6.4 \\
13 & 66 & 6 & 72 & 8.3 \\
14 & 57 & 5 & 62 & 8 \\
15 & 76 & 13 & 89 & 14.6 \\
\hline
\end{tabular}

Recapitulando podemos sacar en claro con el experimento realizado lo siguiente:

- La población métrica de los debris en réplicas de talla presenta una curva característica descendente en donde a menor longitud mayor número de restos hasta tamaños en torno a $8 \mathrm{~mm}$. A partir de aquí bajan hasta ser esporádicos.

- Existe un rango entre los diferentes tipos de debris producidos en réplicas de retoque. Para el caso del sílex utilizado en La Carihuela proponemos como rango normal $6.4 \%-14.6 \%$.

- Ni la materia prima ni el tipo de retoque condicionan de forma clara dicha relación.

\section{DISCUSIÓN}

El estudio de los yacimientos puede hacerse desde variados puntos de vista, pero para llevar a cabo cualquiera de ellos hay que conocer muy bien los procesos de formación que ha sufrido el yacimiento. Si no se realizan estos tipos de estudios en los yacimientos, nuestras hipótesis y resultados variarán cuando se apliquen, haciendo perder mucho esfuerzo y obligándonos a cambiar nuestros resultados con nuevas revisiones (Dibble et alli, 1997).

Los conjuntos arqueológicos sufren transformaciones desde el momento que son abandonados por los humanos hasta que son encontrados. Durante este periodo de tiempo sufren numerosas alteraciones. Este problema es estudiado gracias a muchos instrumentos que nos ayudan más a controlar y no tanto a identificar los diferentes procesos postdeposiciona- 
les. Esto es debido a que cuando sólo actúa un proceso postdeposicional es relativamente sencillo identificarlo, pero cuando actúan más de uno (lo más común en el registro arqueológico) resulta casi imposible (Schiffer, 1987). En este tipo de estudios para conocer la formación de los yacimientos debemos usar todos los instrumentos que tenemos en nuestra mano. La experimentación en algunos procesos puede ser de gran utilidad. Así por ejemplo, existe variada información sobre algunos aspectos postdeposicionales como el pisoteo, sin duda el proceso con el que más se ha experimentado, bien sobre conjuntos líticos (Courtin \& Villa, 1982; McBrearty et al., 1998; Villa \& Courtin, 1983) u óseos (Gifford-Gonzalez et al, 1985), o sobre procesos naturales como la acción eólica (Lancaster, 1986) o perturbación por fauna (Erlandson, 1984).

Este trabajo quiere ser una aproximación a esta problemática. La utilización del debris como agente tafonómico se presenta como acertada sobre todo teniendo en cuenta la facilidad con la que es alterada por causas naturales. Los resultados experimentales que aquí se presentan permiten conocer de una forma real la distribución de las industrias líticas en laboratorio. Estos datos pueden ser traspuestos al registro fósil para poder llegar a discernir posibles procesos postdeposicionales. Sin embargo no debemos perder de vista la globalidad de los datos que nos ofrece el yacimiento, ya que con un estudio completo de todos ellos se llegará a una explicación acertada del mismo.

A la vista de los párrafos anteriores podemos sacar como conclusiones los siguientes aspectos:

1. Los conjuntos líticos estudiados presentan en sus restos menores de $2 \mathrm{~cm}$ una curva característica descendente. En ella se observa un pico máximo en el intervalo menor representado $2-3 \mathrm{~mm}$ y un descenso rápido de la misma 6-8 mm desde la que se presenta constante en valores muy bajos o nulos. Esta distribución se encuentra en otros experimentos similares (Baumler y Downum, 1989; Schick, 1986, 1987) aunque los tipos de materias primas sean diferentes. Por ello esta distribución de la fracción menor de las industrias líticas puede permitir reconocer un conjunto no modificado por procesos naturales, ya que si así fuese la distribución de estos restos presentaría otra dinámica.

2. Dentro de los conjuntos líticos, la fracción debris es la única que no se ve sometida a modificaciones postdeposicionales de origen cultural, excepto casos puntuales, ya que los humanos no los modifican una vez depositados. Al contrario que las lascas, núcleos y demás artefactos de la cadena operativa que pueden ser transportados. De aquí viene su valor como indicador natural de fenómenos de este tipo. 
3. La materia prima es la variable a priori más importante en los experimentos que sobre debris se han realizado y puede hacer variar las conclusiones de los mismos. Se ha comprobado que esta no es del todo condicionante en el experimento realizado, pero hay que recalcar que los dos grupos de materias primas eran de gran calidad para la talla. Sería conveniente la réplica en otros tipos de sílex y materias primas.

4. Proponemos como porcentaje superior en una relación entre debris de lascado y astillado el $15 \%$, aunque hay que señalar que no es más que una frontera aproximativa y que pueden existir conjuntos que lo superen, como asi ha sucedido en el propio experimento. De hecho en experimentos realizados por otros investigadores (Baumler \& Downum, 1989) la talla de los núcleos arroja porcentajes mucho mayores a los de retoque (que son similares a los obtenidos por nosotros) estando en torno al $30 \%$.

\section{BIBLIOGRAFÍA}

AlLARD, M. (1993): Remontage lithique exceptionnel dans le Solutreen Inferieur des Peyrugues (Orniac, Lot). Palèo, 5: 179-191.

BAUMLER, M.F. (1985): On the interpretation of chipping debris concentrations in the archaeological record. Lithic Technology, 14(3): 120-125.

BAUMLER, M.F. \& DOWNUM, C.E. (1989): Between micro and macro: a study in the interpretation of small-sized lithic debitage. Experiments in lithic technology. (Amick, E., ed.). B.A.R. International Series, 528: 101-116.

BEHM, J.A. (1983): Flake concentrations: Distinguishing between flintworking activity areas and secondary deposits. Lithic Technology, 12(1): 9-16.

Bernaldo de Quirós, F; Cabrera, V.; Cacho, C. \& VeGa, L.G. (1981): Proyecto de análisis técnico para las industrias líticas. Trabajos de Prehistoria, 38: 9-37.

COURTIN, J. \& VILLA, P. (1982): Une expérience de piétinement. B.S.P.F., 79(4): 117-123.

DibBLE, H.L. (1995): Middle Paleolithic Scraper Reduction: Background, Clarification, and Review of the Evidence to Date. Journal of Archaeological Method and Theory, 2(4): 299368.

Dibble, H.L.; Chase, Ph.G.; McPherron, S.P. \& Tuffreau, A. (1997): Testing the reality of a Aliving floor with archaeological data. American Antiquity, 62(2): 629-651.

ERLANDSON, J.M. (1984): A case study in faunalturbation: Delineating the effects of the burrowing pocket gopher on the distribution of archaeological materials. American Antiquity, 49(4): 785-790.

FLADMARK, K.R. (1982): Microdebitage analysis: Initial considerations. Journal of Archaeological Science, 9: 205-220.

Freeman, L.G.; Gonzalez Echegaray, J.; Pokines, J.; Stettler, H. \& Krupa, M. (1998): Tamisage ultra fin et récupération de l'outillage: observations réalisées a El Juyo (Espagne Cantabrique). L'Antrhopologie, 102 (1): 35-44.

Gifford-Gonzalez, D.P.; Damrosh, D.B.; Damrosh, D.R.; Pryor, J. \& Thunen, R.L. (1985): The third dimension in site structure: an experiment in trampling and vertical dispersal. American Antiquity, 50(4): 803-818.

Henry, D.O.; Haynes, C.V. \& Bradley, B. (1976): Quantitative variations in flaked stone debitage. Plains Antrhopologist, 21: 57-61.

HuLL, K.L. (1987): Identification of cultural site formation processes through microdebitage analysis. American Antiquity, 54(2): 772-783.

LANCASTER, J. (1986): Wind Action of Stone Artifacts: an Experiment in Site Modification. Journal of Field Archaeology, 13: 359-363. 
McBrearty, S.; Bishop, L.; Plummer, T.; Dewar, R. \& Conard, N. (1998): Tools underfoot: Human trampling as an agent of lithic artifact edge modification. American Antiquity, 63(1): 108-129.

Patterson, L.W. \& Sollberger, J.B. (1978): Replication and classification of small siza lithic debitage. Plains Antrhopologist, 23: 103-112.

Roebroeks, W.: Kolen, J.; van Poecke, M. \& VAN GiJn, A. (1997): A Site J: An Early Weichselian (Middle Palaeolithic) Flint Scatter at Maastricht-Belvedere, The Netherlands. Palèo, 9: 143172.

SCHICK, K.D. (1986): Stone Age Sites in the Making: Experiments in the Formation and Transformation of Archaeological Ocurrences. B.A.R. International Series, 319. Oxford.

SCHICK, K.D. (1987): Experimentally-derived criteria for assessing hydrologic disturbance of archaeological sites. En: Nash, D.T. \& Petraglia, M.D. (eds.): Natural Formation Processes and the Archaeological Record. B.A.R. International Series, 352: 86-107.

SCHIFFER, M.B. (1983): Toward the identification of formation processes. American Antiquity, 48: $675-706$.

SHOTT, M.J. (1994): Size and form in the analysis of Flake Debris: Review and recent approaches. Journal of Archaeological Method and Theory, 1(1): 69-110.

StEIN, J.K. (1987): Deposits for Archaeologist. Advances in Archaeological Method and Theory, 11: 337-339.

STEIN, J.K. \& TELster, P.A. (1989): Size distributions of artifact classes: combining Macro- and Micro-fractions. Geoarchaeology, 4 (1): 1-30.

Vega Toscano, L.G. (1988): El Paleolítico Medio del Sureste español y Andalucía Oriental. Col. Tesis Doctorales, Univ. Complutense de Madrid, 4 vols, 2.364 págs.

VILLA, P. \& COURTIN, J. (1983): The interpretation of Stratified Sites: a View from Underground. Journal of Archaeological Science, 10: 267-281. 